\title{
Determining need for hospitalisation: Evaluation of the utility of the CRB-65 score in patients with community-acquired pneumonia presenting to an emergency department
}

D M Kabundji, ${ }^{1}$ MB BCh, MSc Med (Emergency Medicine); A Musekiwa, ${ }^{2}$ MSc (Math Statistics); M Mukansi, ${ }^{3}$ MB BCh, FCP (SA); C Feldman, ${ }^{3} \mathrm{MB}$ BCh, DSc, PhD, FRCP, FCP (SA)

${ }^{1}$ Helen Joseph Hospital and Division of Emergency Medicine, Department of Family Medicine, Faculty of Health Sciences, University of the Witwatersrand, Johannesburg, South Africa

${ }^{2}$ Wits Reproductive Health and HIV Institute, Faculty of Health Sciences, University of the Witwatersrand, Johannesburg, South Africa

${ }^{3}$ Division of Pulmonology, Department of Internal Medicine, Faculty of Health Sciences, University of the Witwatersrand, Johannesburg, South Africa

Corresponding author: D M Kabundji (jdmulombe@yahoo.fr)

\begin{abstract}
Background. The CRB-65 severity of illness score, used for assessing patients with community-acquired pneumonia (CAP), may be of particular benefit in resource-constrained areas, since it relies purely on clinical parameters.

Objective. To assess the potential accuracy of the CRB-65 score when used in deciding whether to hospitalise patients with CAP presenting to an emergency department (ED).

Methods. Prospective, observational study in an academic hospital in Johannesburg, South Africa. Data from adult patients with radiologically confirmed CAP were analysed.

Results. Overall, 152 patients were enrolled (79 females, 73 males; median age 36.5 years). Several diverse criteria had been used by the ED doctors in admission decisions, while the CRB-65 score had been used in only $3 / 152$ patients (1.6\%). Overall, 68/152 patients (44.7\%) had been managed as inpatients and 84/152 (55.3\%) as outpatients. If the CRB-65 had been used as the sole criterion for site-of-care decisions, $107 / 152$ patients (70.4\%) would potentially have been managed as outpatients and 45/152 (29.6\%) as inpatients. Achieving a stable clinical condition took longer $(p=0.037)$ and mortality was higher $(p<0.001)$ in patients with higher than lower CRB-65 scores. All five patients who died were inpatients. Of these, three $(60.0 \%)$ would have been classified by the CRB-65 as having an intermediate mortality risk and two (40.0\%) as having a high mortality risk.

Conclusions. This study demonstrates the utility of the CRB-65 score in accurately determining the need for admission of patients with CAP presenting to an ED in a resource-constrained environment.

S Afr Med J 2014;104(11):769-772. DOI:10.7196/SAMJ.8150
\end{abstract}

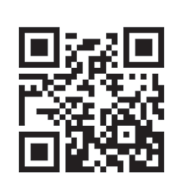

Despite recent advances in the management of community-acquired pneumonia (CAP), it remains a common and potentially lethal infectious disease. CAP mortality is variable, depending on the site of care. It is $<1 \%$ in the outpatient setting, about $5-15 \%$ in inpatients not requiring intensive care unit (ICU) care, up to $25 \%$ in intubated patients, and nearly $50 \%$ in ICU patients requiring vasopressors. $^{[1-4]}$ Determination of disease severity is crucial in the assessment and management of patients with CAP in the emergency department (ED), since it guides various interventions and decisions, including the optimal site of care (i.e. need for hospital or ICU admission or suitability for home care).

Several tools have been developed to assist in the prediction of severity of CAP, including the CRB-65 score. ${ }^{[3]}$ This scoring system was developed from the CURB-65 score and derived from the British Thoracic Society rule, but is simpler to use. It has been recommended for use in the community setting in the UK, and has an accuracy similar to that of the CURB-65 score and the Pneumonia Severity Index (PSI) ${ }^{[1,3,5]}$ However, since it does not require measurement of the blood urea level, it may be of particular value in areas with limited resources.

We wished to assess the potential accuracy of the CRB-65 score used to determine the need for admission to hospital of patients with CAP presenting to the ED at Helen Joseph Hospital (HJH), Johannesburg, South Africa (SA), in an area with a high prevalence of HIV infection.

\section{Materials and methods \\ Study design and population}

This was a prospective, observational, hospital-based study of a consecutive sample of 152 adult patients aged $\geq 18$ years with CAP seen in the ED at HJH between February 2011 and April 2011. It was purely an observational study and the researchers did not play any role in clinical management of the patients, including decisions regarding severity of illness or need for hospital admission. Once the ED doctors had diagnosed a patient as having CAP and personally managed the case (including determining, by whatever criteria they used, whether the patient needed to be admitted to hospital or not), they informed the primary study investigator (DMK) about the patient. After obtaining written informed consent from the patient, DMK evaluated each case for purposes of the study, in the first instance confirming that the patient actually had CAP.

For the purposes of the study the following criteria were used for the definition of CAP, as described previously: ${ }^{[6]}$ two or more of the following: altered breath sounds and/or signs of lung consolidation, fever, rigors, sweats and cough, with or without sputum production, 
pleuritic chest pain, cyanosis, shortness of breath and tachypnoea, together with radiological confirmation of the diagnosis of pneumonia. Chest radiographs (CXRs) were initially evaluated by the attending ED doctors or registrars, and confirmed for study purposes as demonstrating pneumonia by DMK. Excluded were cases of suspected or confirmed aspiration pneumonia, chemical pneumonitis, Pneumocystis jirovecii pneumonia and pulmonary tuberculosis. Patients with any acute or active comorbid illness such as diabetes mellitus, renal failure, cardiac failure or end-stage AIDS were similarly excluded.

Thereafter, DMK found out from the ED doctors what criteria they had used to assess the severity of illness and/or determine the need or not for hospital admission of the patients. The CRB-65 severity of illness score was then evaluated in each CAP study patient by the primary researcher. In this scoring system, one point is assigned for each of the following parameters, if present: confusion, respiratory rate (RR) $\geq 30 / \mathrm{min}$, systolic blood pressure (BP) $<90 \mathrm{mmHg}$ and/or diastolic $\mathrm{PB} \leq 60 \mathrm{mmHg}$, and age $\geq 65$ years..$^{[3,5]}$ The abbreviated mental test, modified for SA conditions (AMTMSA), was used for objective assessment of the presence/ absence of confusion. ${ }^{[3,4,7]}$ Confusion was further defined as an AMTMSA score of $\geq 8$ or presence of new disorientation for place or time..$^{[7-9]}$ It has previously been suggested that patients with a CRB-65 score of 0 are at low risk of mortality and may be suitable for management as outpatients. Patients with a score of 1 or 2 are at intermediate risk of mortality and should be considered for hospital-supervised treatment, while those with scores of 3 or 4 are at high risk of mortality and may require high care or ICU care. ${ }^{[3,5]}$ The study patients were therefore classified into low, moderate and high mortality risk groups.

DMK documented demographic and clinical features, including age, gender, the AMTMSA score, systolic and diastolic blood pressure, heart rate, respiratory rate, body temperature, site of care (outpatient or inpatient) and outcome. These parameters were used to determine the CRB-65 score, as well as the time to clinical stability and the mortality rate. Time to clinical stability was determined according to a validated rule, described previously, that defined clinical stability as the first day on which most of the following criteria were simultaneously achieved: systolic BP $\geq 90 \mathrm{mmHg}$; RR $\leq 24$ / min; heart rate $\leq 100 \mathrm{bpm}$, oxygen saturation (on room air) $\geq 92 \%$, temperature $\leq 37.2^{\circ} \mathrm{C}$, ability to tolerate oral intake, and baseline mental status. ${ }^{[10]}$ Admission day was day 0 , with the second admission day as day 1 and so on. Patients who were treated as outpatients were personally contacted telephonically 2 weeks after the ED visit, with prior consent, to determine their outcome.

The numbers of patients admitted and discharged by the ED physicians were compared with the numbers who would have been admitted or discharged if the CRB-65 score had been used in this decision-making process. Furthermore, the CRB-65 scores were evaluated in relation to the time to clinical stability among the patients who were admitted, and to the outcome of all cases, including those treated at home. These outcome variables were used to evaluate the potential accuracy, and hence the utility, of the CRB-65 score.

\section{Statistical methods}

For the analysis of the data, descriptive statistics were summarised using frequencies and cross-tabulations. For non-normally distributed continuous variables, medians and their associated ranges were calculated and reported. Associations between categorical outcomes were formally tested using the $\chi^{2}$ test and Fisher's exact test. The latter was used when the expected numbers of subjects in the cells were less than 5 . Results were presented using $p$-values. Throughout the analysis, two-sided statistical tests were used at the $5 \%$ level of significance. STATA 11.0 was used in the analysis of the data.

\section{Results}

A total of 152 patients were enrolled into the study. There were 79 females $(52.0 \%)$ and 73 males (48.0\%), age range $20-87$ years (median 36.5). The majority of the patients (98.0\%) had a normal AMTMSA score $(10 / 10)$. The median systolic blood pressure was $114 \mathrm{mmHg}$ (range 86 - 172), diastolic blood pressure $72 \mathrm{mmHg}(35$ $100)$, heart rate $103 \mathrm{bpm}(58-158), \mathrm{RR}$ $23.5 / \mathrm{min}(14-38)$ and temperature $37.8^{\circ} \mathrm{C}$ (36 - 41).

As shown in Table 1, the most common criterion used by the HJH ED doctors to decide whether patients should be admitted to hospital or not was the appearance of the CXR (41.5\%), followed by the haemodynamic parameters of the patients (25.9\%) and thereafter, somewhat less frequently, various other parameters. The CRB-65 score was used in only $1.6 \%$ of cases.

On the basis of these criteria, $68 / 152$ (44.7\%) of all the enrolled patients had been managed in hospital by the ED physicians, with the remaining $84 / 152(55.3 \%)$ treated as outpatients. Had the CRB-65 score been the sole criterion on which this decision had been based, 107/152 patients (70.4\%) would potentially have been managed as outpatients and 45/152 (29.6\%) in hospital.

Table 2 shows the association between the CRB-65 score and the time to clinical
Table 1. Criteria used for decisions regarding admission or discharge of CAP patients

\begin{tabular}{|c|c|c|c|}
\hline Criterion & Admitted $n(\%)$ & Discharged $n(\%)$ & Total $n(\%)$ \\
\hline CXR & $47(49.0)$ & $33(34.0)$ & $80(41.5)$ \\
\hline Haem. para. & $3(3.0)$ & $47(48.0)$ & $50(25.9)$ \\
\hline Blood & $6(6.0)$ & $15(15.0)$ & $21(10.9)$ \\
\hline $\mathrm{O}_{2}$ sat. & $20(21.0)$ & - & $20(10.4)$ \\
\hline Fever & $5(5.0)$ & _ & $5(2.6)$ \\
\hline CURB-65 & $2(2.0)$ & $2(2.0)$ & $4(2.1)$ \\
\hline CRB-65 & $2(2.0)$ & $1(1.0)$ & $3(1.6)$ \\
\hline Adv. RVD & $2(2.0)$ & _ & $2(1.0)$ \\
\hline Tachycardia & $2(2.0)$ & _ & $2(1.0)$ \\
\hline $\mathrm{RD}$ & $2(2.0)$ & - & $2(1.0)$ \\
\hline IV antibiotic & $1(1.0)$ & - & $1(0.5)$ \\
\hline Dehydration & $1(1.0)$ & _ & $1(0.5)$ \\
\hline Ren. dysf. & $1(1.0)$ & - & $1(0.5)$ \\
\hline SOB & $1(1.0)$ & - & $1(0.5)$ \\
\hline Total & $95(100.0)$ & $98(100.0)$ & $193(100)$ \\
\hline
\end{tabular}




$\begin{aligned} & \text { Table 2. Association between time to clinical stability and CRB-65 scores among } \\
& \text { the CAP patients }\end{aligned}$
\begin{tabular}{lllll}
\multicolumn{4}{c}{ Time to clinical stability (days) } \\
\cline { 2 - 4 } CRB-65 score & $\mathbf{1}$ & $\mathbf{2}$ & $\mathbf{3}$ & Total, $\boldsymbol{n}$ \\
\hline 0 & $16(47.0)$ & $17(50.0)$ & $1(3.0)$ & 34 \\
$1-2$ & $6(21.5)$ & $16(57.0)$ & $6(21.5)$ & 28 \\
$3-4$ & - & $1(100.0)$ & - & 1 \\
Total & $22(35.0)$ & $34(54.0)$ & $7(11.0)$ & 63 \\
CAP = community-acquired pneumonia. & & & &
\end{tabular}

Table 3. CRB-65 scores in patients stratified according to outcome (alive/dead)

\begin{tabular}{llll}
\hline CRB-65 score & Alive, $\boldsymbol{n}$ (\%) & Dead, $\boldsymbol{n}$ (\%) & Total, $\boldsymbol{n}$ \\
\hline 0 & $107(100.0)$ & - & 107 \\
$1-2$ & $39(93.0)$ & $3(7.0)$ & 42 \\
$3-4$ & $1(33.0)$ & $2(67.0)$ & 3 \\
Total & $147(97.0)$ & $5(3.0)$ & 152
\end{tabular}

stability. There was a significantly shorter time to clinical stability in patients with a lower CRB-65 score (Fisher's exact test $p=0.037)$. The total of 63 in-hospital patients in the table excludes the five deaths that occurred in the overall total of 68 in-hospital patients. These five patients did not reach clinical stability at any time during their hospital stay and therefore could not be used in the assessment of this parameter.

Table 3 shows the association between mortality and the CRB-65 score in the CAP patients. There were no deaths among the outpatients, but five of the in-hospital patients died. Three of these five patients would have been classified by the CRB- 65 score as having an intermediate mortality risk and two as having a high mortality risk, which would have required that they be admitted. Patients with a higher CRB-65 score were at a significantly higher risk of death than patients with a lower CRB-65 score (Fisher's exact test $p<0.001$ ).

\section{Discussion}

\section{Main study findings}

The main findings of this study, which we believe is the first in a resource-constrained environment, were as follows: (i) the CRB-65 score was used very infrequently by the ED doctors at this hospital; (ii) had the CRB-65 score been used as the criterion for hospital admission, far fewer patients would have been admitted (all the additional patients who were admitted to hospital having had a good outcome), (iii) all the discharged patients had a CRB-65 score confirming that they could indeed be considered for treatment safely at home (notably, all survived); (iv) the patients admitted to hospital with a lower CRB-65 score had a shorter time to clinical stability and a lower mortality compared with patients with a higher CRB-65 score; and $(v)$ all the patients who ultimately died would have been admitted on the basis of their CRB-65 scores.

\section{Interpretation of findings in relation to previously published work}

The CRB-65 score was used very infrequently by the ED doctors, being utilised in only $3 / 152$ cases (1.6\%), while the CURB-65 score was utilised in only $4 / 152$ cases $(2.6 \%)$. Surprisingly, there have been very few studies investigating the use of severity of illness scoring systems by ED clinicians, but at least one study documented low utilisation and compliance with a validated ED triage system. ${ }^{[11]}$ Furthermore, even when severity of illness scoring systems are used for assessment of patients with pneumonia in the ED, ED providers often do not rely on them for decision-making regarding hospitalisation. ${ }^{[12]}$ However, we did not address the question of why the doctors did not use the scoring system more frequently.

The CXR was the most frequently used criterion used by the ED doctors for admitting CAP patients to hospital for treatment, whereas haemodynamic criteria were most commonly used for discharge decisions. The specific CXR criteria that encouraged HJH ED doctors to admit patients were not specifically investigated in the current study.
However, when we subsequently reviewed the CXR findings of all the patients (data not shown), we noted that the most common radiographic features among the patients who had been admitted were bilateral infiltration, multilobar consolidation and significant pleural effusion. Interestingly, in the study by Espana et al. ${ }^{[13]}$ multilobar/ bilateral lung involvement on the CXR and abnormal haemodynamic parameters (systolic BP <90 mmHg, RR >30/min and altered mental status) were among eight independent predictive factors for severe CAP.

Had the CRB-65 score been used, fewer patients would have been admitted to hospital. ED doctors admitted 68/152 patients (44.7\%) and discharged 84/152 (55.3\%), whereas had the CRB-65 score been used for admission decisions, a total of $107 / 152$ patients $(70.4 \%$ - all classified as at low mortality risk) would potentially have been managed as outpatients, while $45 / 152$ $(29.6 \%$ - 42 classified as at intermediate mortality risk and three as at high mortality risk) would have been managed as inpatients. Similarly, in the study described above documenting that ED providers did not rely on the PSI for determining the initial site of care for patients with CAP, many lowrisk cases $(258 / 689$; 37.4\%) were admitted to hospital. ${ }^{[12]}$ Evidence suggests that ED physicians tend to overestimate the risk of death among patients with CAP, consequently leading to hospitalisation of many patients at low risk of mortality. Importantly, in the current study the outcome of all these additional low-risk patients who had been hospitalised was good, and there were no deaths among them. It is clear that this may have considerable cost implications, as demonstrated in many studies. In their study of the cost of treating patients with CAP, Lave et al. ${ }^{[14]}$ concluded that hospital admission of low-risk patients with CAP is far more costly than outpatient treatment. A documented example given was \$264 for outpatients and \$7 500 for inpatients (including hospital and physician care and follow-up care). In a study of preferences of home v. hospital care among low-risk patients with CAP, Coley et al. ${ }^{[15]}$ came to a similar conclusion.

Among the patients admitted to hospital, those with a lower CRB-65 score had a significantly shorter time to clinical stability $(p=0.037)$ and lower mortality. All the deaths were of inpatients: $3 / 5(60.0 \%)$ would have been classified by the CRB- 65 score as having an intermediate mortality risk, with the remaining $2 / 5(40.0 \%)$ having a high mortality risk. If the CRB-65 score had been used as the 
sole criterion for hospital admission, all the deaths would have occurred among patients who would have been assessed as needing hospital, and possibly even high-care or ICU, admission. The CRB-65 score would therefore have appeared to perform well, being able to predict the time to clinical stability and the risk of death for hospitalised patients with CAP. Others have arrived at the same conclusion. For example, in their study of predictive accuracy of the PSI versus the CRB-65 for time to clinical stability, Arnold et al. ${ }^{[16]}$ found that the CRB-65 score was a powerful yet simple tool for predicting time to clinical stability and death among patients with CAP. In a study of 338406 patients with CAP from Germany, Ewig et al..$^{[17]}$ came to a similar conclusion.

As international studies have done, our study therefore showed that the CRB-65 severity of illness score, which is based exclusively on three bedside clinical signs and age, appears to accurately predict the time to clinical stability and the risk of death in patients with CAP. Importantly, this scoring tool worked well in a resource-constrained environment that is also an area of high HIV prevalence.

\section{Limitations and strengths of the study}

The study has limitations. While undertaken in SA, a country with a high prevalence of HIV and resource constraints, it encompassed only one institution. The findings may therefore not be generalisable to other countries or other institutions. Furthermore, the ethnic origin of the patients, their socioeconomic status and home circumstances, and their habits (with regard to alcohol consumption, drug use and cigarette smoking) were not recorded. It is possible that these factors may have impacted on our findings.

The study has strengths. It was a prospective investigation, and therefore all the information that was required for the study analysis was collected. It is also the first study we are aware of that used the CRB-65 score in a resource-constrained environment.

\section{Conclusion}

In conclusion, this study demonstrates the potential ability of the CRB-65 severity of illness scoring system to assist ED physicians with decisions regarding the optimal site of care of patients with $\mathrm{CAP}$ in a resource-constrained environment. Further studies are required, in particular an interventional study in which the CRB65 score is implemented for decision-making in some patients, and its performance compared with cases in which decision-making is based on the usual standard of care, whatever that may be. Lastly, it is important to remember that severity of illness scoring systems are a guide and cannot take the place of sound clinical judgement.

Acknowledgements. This study formed the basis of DMK's research report for the MSc Med degree in the specialty of Emergency Medicine from the
University of the Witwatersrand. The authors thank the study participants, Helen Joseph Hospital and the University of the Witwatersrand staff who assisted in this study.

Authors' contributions. DMK planned the study, wrote the protocol, performed data processing, evaluated and interpreted the results and wrote the article. AM performed the statistical analysis and assisted in the writing up of the statistical methods and results. He also assisted in the writing of the article. MM assisted in the writing of the protocol and the article. CF conceived the study, and assisted in the writing up of the protocol, in evaluation and interpretation of the results, and in the writing of the article.

Funding. CF is supported by the National Research Foundation of South Africa.

\section{References}

1. Restrepo MI, Anzueto A. Severe community-acquired pneumonia. Infect Dis North Am 2009;23(3):503-520. [http://dx.doi.org/10.1016/j.idc.2009.04.003]

2. Moran G], Talan DA, Abrahamian FM. Diagnosis and management of pneumonia in the emergency department. Infect Dis Clin North Am 2008;22(1):53-72. [http://dx.doi.org/10.1016/j.idc.2007.10.003]

3. Angus DC, Marrie TJ, Obrosky DS, et al. Severe community-acquired pneumonia: Use of intensive care services and evaluation of American and British Thoracic Society diagnostic criteria. Am J Respir Crit Care Med 2002;166(5):717-723. [http://dx.doi.org/10.1164/rccm.2102084]

4. Rodriguez A, Mendia A, Sirvent JM, et al. Combination antibiotic therapy improves survival in patients with community-acquired pneumonia and shock. Crit Care Med 2007;35(6):1493-1498. http://dx.doi.org/10.1097/01.CCM.0000266755.75844.05]

5. Feldman C, Brink AJ, Richards GA, et al. Management of community-acquired pneumonia in adults. South Afr J Epidemiol Infect 2008;23(2):31-42. [http://dx.doi.org/10.1016/S1473-3099(07)70242-6] RD Bulletin. June 2007, volume 14 issue 5. www.ich.ucl.ac.uk/bulletin (accessed 27 December 2013)

7. Moran G. Approaches to treatment of community-acquired pneumonia in the emergency department and the appropriate role of fluoroquinolones. J Emerg Med 2006;30(4):377-387. [http://dx.doi. org/10.1016/j.jemermed.2005.07.009]

8. Hodkinson HM. Evaluations of a mental test score for assessment of mental impairment in the elderly. Age Ageing1972;1(4):233-8. [http://dx.doi.org/10.1093/ageing/1.4.233]

Wikipedia. Abbreviated mental state score. 2009. http://en.wikipedia.org/wiki/abbreviated_mental_ test_score\#cite_note-hodkinson72-0 (accessed 22 December 2013).

10. Menendez R, Torres A, Rodriguez de Castro F, et al. Reaching stability in community-acquired pneumonia: The effects of the severity of disease, treatment, and the characteristics of patients. Clin Infect Dis 2004;39(12):1783-1790. [http://dx.doi.org/10.1086/426028]

11. Christensen D, Jensen NM, Maaloe R, et al. Low compliance with a validated system for emergency department triage. Dan Med Bull 2011;58(6):A4294.

12. Aujesky D, McCausland JB, Whittle J, et al. Reasons why emergency department providers do not rely on pneumonia severity index to determine the initial site of treatment for patients with pneumonia. on pneumonia severity index to determine the initial site of treatment for
Clin Infect Dis 2009;49(10):e100-e108. [http://dx.doi.org/10.1086/644741]

13. Espana PP, Capelastegui A, Gorordo I, et al. Development and validation of a clinical predictive rule for severe community-acquired pneumonia. Am J Respir Crit Care Med 2006;174(11):1249-1256. [http:// severe community-acquired pneumonia.
dx.doi.org/10.1164/rccm.200602-1770C]

14. Lave JR, Lin CJ, Hughes-Cromwish P, et al. The cost of treating patients with communityacquired pneumonia. Semin Respir Crit Care Med 1999;20(3):189-198. [http://dx.doi. acquired pneumonia. Sen
org/10.1055/s-2007-1021315]

15. Coley CM, Li YH, Medsger AR, et al. Preferences for home vs hospital care among low-risk patient with community-acquired pneumonia. Arch Intern Med 1996;156(14):1565-1571. [http://dx.doi.
wer org/10.1001/archinte.1996.00440130115012]

16. Arnold FW, Brock GN, Peyrani P, et al. Predictive accuracy of the pneumonia severity index vs CRB65 for time to clinical stability: Results from the Community-Acquired Pneumonia Organization 65 for time to clinical stability: Results from the Community-Acquired Pneumonia Organization
(CAPO) international cohort study. Resp Med 2010;104(11):1736-1743. [http://dx.doi.org/10.1016/j. (CAPO) internatio

17. Ewig S, Birkner N, Strauss R, et al. New perspectives on community-acquired pneumonia in 388406 patients. Results from a nationwide mandatory performance measurement programme in healthcare patients. Results from a nationwide mandatory performance measurement program
quality. Thorax 2009;64(12):1062-1069. [http://dx.doi.org/10.1136/thx.2008.109785]

Accepted 12 May 2014 